\title{
The homologous recombination protein RAD51 is a promising therapeutic target for cervical carcinoma
}

\author{
QIAN CHEN, DONGGE CAI, MU LI and XIAOLING WU \\ Department of Obstetrics and Gynecology, The Second Affiliated Hospital of \\ Xi'an Jiaotong University, Xi'an, Shaanxi 710004, P.R. China
}

Received January 7, 2017; Accepted June 6, 2017

DOI: $10.3892 /$ or.2017.5724

\begin{abstract}
RAD51 is one of the pivotal enzymes for DNA double-strand break (DSB) repair by the homologous recombination (HR) pathway, which implies it as a promising and novel target for cancer therapy. Recent findings have indicated RAD51 protein is overexpressed in a variety of tumors. The high-expression of RAD51 is related to poor prognosis. RAD51 is involved in the repair of DNA damage and the generation of genetic diversity by an evolutionarily conserved mechanism. However, the exact mechanism of RAD51 in the progression of cervical cancer remains unclear. RI-1 is a small molecule that inhibits the central recombination protein RAD51. In this study, we found that RAD51 was highly expressed in invasive squamous cervical cancer (SCC). The administration of RI-1 inhibited cell growth in vitro and reduced growth of tumor xenografts in vivo with cervical cancer cells (HeLa and $\mathrm{SiHa}$ ). Further investigation suggested that RAD51 protein significantly promoted the cell cycle transition from the G0/G1 to $S$ phase. In addition, the inhibition of RAD51 reduced the level of the cell cycle related protein cyclin D1, but increased the levels of $\mathrm{p} 21 \mathrm{mRNA}$ and protein. As a DNA DSB repair enzyme, the expression of RAD51 in tumor cells possibly affects their sensitivity to anti-cancer agents. Additionally, in experiments using cisplatin and ionizing radiation, RI-1 treated cervical cancer cells, HeLa and $\mathrm{SiHa}$, were sensitized to a greater extent than the untreated control. Thus, HR inhibition of RAD51 may provide yet another mechanism of therapeutic target for the chemosensitization and radiosensitization of cervical cancer with RI-1. Collectively, our data demonstrated for the first time that inhibition of RAD51 suppressed the cervical cancer cell proliferation and the growth of cervical cancer xenografts by attenuating cell cycle transition, which could be a functional link between RAD51 and cyclin D1 and p21.
\end{abstract}

Correspondence to: Dr Xiaoling Wu, Department of Obstetrics and Gynecology, The Second Affiliated Hospital of Xi'an Jiaotong University, 157 Xiwu Road, Xi'an, Shaanxi 710004, P.R. China E-mail: wxlxjtu07@stu.xjtu.edu.cn

Key words: RAD51, cervical cancer, proliferation, cyclin D1, p21, chemosensitivity, radiosensitivity

\section{Introduction}

Worldwide, cervical cancer is the second most common malignancy after breast cancer, and remains a leading cause of cancer-related death among females in developing countries. There were an estimated 527,600 new cervical cancer cases and 265,700 deaths worldwide in 2012 (1). Currently, surgery, platinum based chemotherapy and radiotherapy play important roles for cervical cancer treatment. Nevertheless, acquired resistance to platinum and radiation is considered a main element for tumor relapse and metastasis. Therefore, to develop more effective therapeutic strategies and in-depth research into the molecular and biologic mechanisms of oncogenesis for cervical cancer is critical. Although the development of cervical cancer is intimately associated with high-risk human papillomavirus (HPV) infection (2), not all patients infected with HPV ultimately develop cervical cancer. Thus, various molecular dysfunction, including the activation of oncogenes and inactivation of suppressor genes, are also essential for cervical cancer development $(3,4)$.

The RAD51 gene is homologous to the E. coli RecA and yeast $R A D 51$ genes, which are involved in the repair of DNA double-strand breaks and also play important roles in recombination repair and various SOS responses to DNA damage by $\gamma$-irradiation and alkylating reagents (5). RAD51 plays a role in several cellular processes, including genomic integrity, cell cycle regulation, apoptosis and tumor formation. RAD51 is overexpressed in a variety types of tumors, including cervical cancer (6), non-small cell lung cancer (7), breast cancer (8), ovarian cancers (9), pancreatic cancer (10), melanoma and glioblastoma (11). The overexpression of RAD51 causes improper and hyper-recombination, namely contributing to genomic instability and genetic diversity, which might drive regular cells towards neoplastic transformation or further contribute to cancer progression and metastasis $(12,13)$. Additionally, in Fanconi anemia-like patients, phenotype-derived mutation in $R A D 51$ plays a role in protection of DNA during the course of ICL repair, which is independent of RAD51's ability to maintain genomic integrity (14). Accumulating evidence has indicated that $R A D 51$ not only is involved in the progression of carcinogenesis, but also plays a part in resistance to anticancer treatments $(16,17)$. The RAD51 protein is a specific HR-related target for cancer therapy. Treatment of leukemia, prostate cancer, pancreatic adenocarcinoma, lung carcinoma 
and glioma cells with imatinib can decrease RAD51 expression and sensitize them to experimental chemotherapy and radiotherapy in vitro and in vivo $(17,18)$.

The molecular mechanisms of RAD51 in cervical carcinoma are largely unclear. In the present study, we demonstrated that RAD51 was overexpressed in cervical carcinoma and examined the effects of the RAD51 inhibitor RI-1 on the proliferation, cell cycle and sensitivity of cervical cancer cells. Budke et al revealed that RI-1 made RAD51 inactive by directly binding covalently to human RAD51 protein at cysteine 319. Accordingly, RI-1 inhibited the formation of subnuclear RAD51 foci in cells in response to DNA damage, without affecting formation of the replication protein A focus (15). Our study suggested that RI-1 largely inhibits the growth of cervical cancer cells in vitro and in vivo by arresting the cell cycle. Moreover, RI-1 decreased resistance to platinum and ionizing radiation. Therefore, in addition to classical function in HR, RAD51 can also regulate the progression of the cell cycle, and thus RI-1 might have promising therapeutic effects against cervical carcinoma.

\section{Materials and methods}

Cell lines and cell culture. Human cervical cancer cell lines (CaSki, C33A, HeLa and SiHa) were purchased from the American Type Culture Collection (ATCC; Manassas, VA, USA). C33A, HeLa and SiHa cells were cultured in Dulbecco's Modified Eagle's Medium (DMEM; Sigma-Aldrich, St. Louis, MO, USA), and CaSki was cultured in RPMI-1640 (Sigma-Aldrich), supplemented with $10 \%$ heat-inactivated fetal bovine serum (FBS; Invitrogen, Carlsbad, CA, USA). All cell lines were maintained at $37^{\circ} \mathrm{C}$ in a humidified $5 \%$ $\mathrm{CO}_{2}$ incubator.

Tissue specimens. The human specimens $(\mathrm{n}=107)$ were collected from patients at the Second Affiliated Hospital of Xi'an Jiaotong University from 2010 to 2015. Of the 107 samples 43 were normal cervical (NC) tissues and 64 were squamous cervical cancer (SCC) tissues. All of the procedures followed approved medical ethics practices. None of the patients had received chemotherapy, immunotherapy or radiotherapy before specimen collection. The histological classifications and clinical staging were done in accordance with the International Federation of Gynecology and Obstetrics classification system.

Immunohistochemistry and RAD51 inhibitor. Formalinfixed, paraffin-embedded tissue sections were analyzed in an immunohistochemical study. After being placed in a $60^{\circ} \mathrm{C}$ incubator overnight, four micrometer-thick sections were deparaffinized with xylene and rehydrated in a series of ethanols. Then the sections were incubated in heat-induced epitope retrieval in citric acid buffer ( $\mathrm{pH}$ 7.0) for 2 min. Endogenous peroxidase was blocked at room temperature using 3\% hydrogen peroxide in methanol for $10 \mathrm{~min}$. Then slides were incubated with mouse monoclonal anti-RAD51 (1:100; Bioscience, Boston, MA, USA) at $4^{\circ} \mathrm{C}$ overnight, followed with inclubation of goat anti-mouse immunoglobulin at room temperature for $30 \mathrm{~min}$. The slides were counterstained stained with hematoxylin (7). RAD51 immunostaining was presented by a semi-quantitative immunoreactivity score (IRS), which (negative 0-3, weak 4-7, strong 8-12) was evaluated by multiplying the values for staining intensity (scored as 0 , no staining; 1 , light brown; 2 , brown; 3 , dark brown) and the values for percentage of positive cells (scored as $0,<10 \%$; $1,10-25 \% ; 2,25-50 \% ; 3,50-75 \% ; 4,>75 \%$ ) in each sample. An overall score of $\leq 3$ was defined as negative and a score of $>3$ as positive. All specimens were evaluated by two pathologists in a blinded manner.

The RAD51 inhibitor RI-1 was purchased from Selleck Chemicals (Houston, TX, USA), according to the manufacturer's instructions. RI-1 was dissolved in DMSO at a stock concentration of $1 \mathrm{mM}$.

Western blot analysis. Exponentially growing cells were harvested and resuspended in PBS pH 7.4 (Gibco). Then the cells were lysed by lysis buffer mixed with protease inhibitor cocktail (Roche, Mannheim, Germany). The lysate was mixed with Laemmli sample buffer containing 5\% 2-mercaptoethanol and boiled for $5 \mathrm{~min}$. Equal amounts of protein extracts were separated on a $10 \%$ SDS/PAGE, blotted onto an activated polyvinylidene difluoride membrane (Millipore, Billerica, MA, USA) and blocked in TBST with 5\% dried milk. Membranes were probed with anti-RAD51 (1:1000, Bioscience), anti-GAPDH (1:1000, Santa Cruz Biotechnology, Santa Cruz, CA, USA), anti-cyclin D1 (1:500, Santa Cruz Biotechnology), anti-p21 (1:500, Santa Cruz Biotechnology) at $4^{\circ} \mathrm{C}$ overnight. Then, they were probed with goat anti-mouse secondary antibodies (Thermo Fisher Scientific, Grand Island, NY, USA) for $1 \mathrm{~h}$.

Cell growth and cell viability assays. Cells were seeded in triplicates at a density of $5 \times 10^{4}$ cells with $2 \mathrm{ml}$ of media into $35-\mathrm{mm}$ tissue culture dishes for 7 days. The numbers of cells were manually counted after harvesting using a hemocytometer under light microscopy every two days. Cell viability assays were performed by applying 3-(4,5-dimethylthiazol-yl)2,5-diphenyl tetrazolium bromide (MTT; Sigma-Aldrich) dye to cells that were seeded in 96-well plates with 800 cells in each well, as described in a standard protocol. Then, the number of live cells was determined by the absorbance at $490 \mathrm{~nm}$ (Bio-Rad, Hercules, CA, USA).

Tumor xenograft experiment. To assess the tumorigenicity in vivo, cells $\left(1 \times 10^{7}\right)$ in the exponential growth phase were collected and were suspended in $200-\mu 1$ phosphate-buffered saline and then injected subcutaneously into the posterior side of 4- to 6-week-old female BALB/c-nude mice purchased from Shanghai SLAC Laboratory Animal Co., Ltd. (Shanghai, China). Xenograft tumor volume (V) was calculated using the length (a) and width (b) by $\mathrm{V}=\mathrm{ab}^{2} / 2$. To study the function of the RAD51 inhibitor RI-1, $5 \mathrm{mg} / \mathrm{kg}$ in $200-\mu 1$ phosphatebuffered saline was injected intraperitoneally every other day into $\mathrm{BALB} / \mathrm{c}$ nude mice $(\mathrm{n}=6)$ when the tumor volume reached $120 \mathrm{~mm}^{3}$. In this analysis, the negative control group $(\mathrm{n}=6)$ received saline. The animal experimental protocols were evaluated and approved by the Animal Care and Use Committee of the Medical College of Xi'an Jiaotong University. The mice were sacrificed and tumors were dissected and weighed at the end of the experiment. 

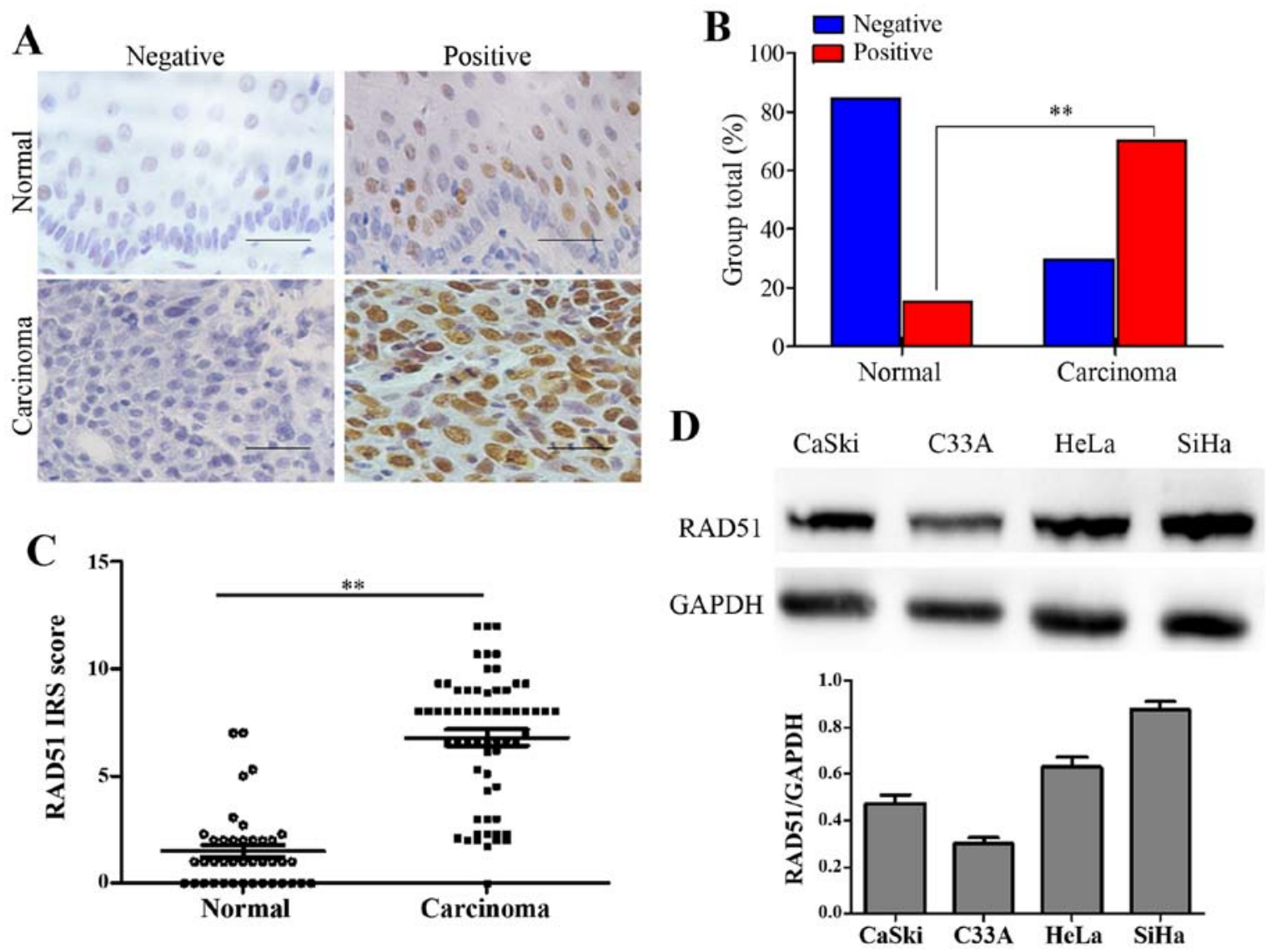

Figure 1. RAD51 expression in normal cervix and cervical carcinoma tissues. (A) Immunohistochemistry results showing RAD51 expression in normal cervix and cervical carcinoma tissues; scale bar, $50 \mu \mathrm{m}$. (B) RAD51 staining is classified into negative and positive, and the percentage of tissues in each group is shown. (C) The IHC scores of RAD51 staining in the normal cervix and carcinoma tissues are shown. (D) The expression of RAD51 in Caski, C33-A, HeLa and SiHa cells was measured by western blotting, the relative expression of RAD51 was calculated based on western blot analyses, GAPDH was used as an internal control. ${ }^{*} \mathrm{P}<0.05,{ }^{* *} \mathrm{P}<0.01$.

Table I. Rad51 expression in tissue specimens.

\begin{tabular}{lcccc}
\hline & & \multicolumn{2}{c}{ Rad51 staining } & \\
\cline { 3 - 4 } Specimens & Total & $\begin{array}{c}\text { Negative, } \\
\text { no. }(\%)\end{array}$ & $\begin{array}{c}\text { Positive, } \\
\text { no. }(\%)\end{array}$ & P-value \\
\hline $\begin{array}{l}\text { Normal } \\
\text { Carcinoma }\end{array}$ & 43 & $37(86.05)$ & $6(13.95)$ & \\
\hline
\end{tabular}

Flow cytometry analysis. Cell cycle analysis was performed using flow cytometry (FACScan; Becton Dickinson, Franklin Lakes, NJ, USA) according to the manufacturer's instructions. Cells were harvested and fixed in $70 \%$ ice-cold ethanol overnight at $4^{\circ} \mathrm{C}$. Thirty minutes before FACS analysis, the samples were washed with PBS, treated with $1 \mathrm{mg} / \mathrm{ml}$ RNase A and then stained with $20 \mu \mathrm{g} / \mathrm{ml}$ propidium iodide (Sigma-Aldrich). Cell cycle distribution was analyzed using a FACSCalibur flow cytometer with Mod-Fit LT software.

Quantitative real-time PCR. Total RNA was extracted from exponentially growing cells with the TRIzol reagent (Invitrogen). Total cDNA was used as a template for PCR amplification. Quantitative real-time PCR was performed using the IQ5 Real-time PCR Detection System (Bio-Rad) in triplicates and the following primers: GAPDH (GCACCGT CAAGGCTGAGAAC and TGGTGAAGACGCCAGTGGA); Cyclin D1 (AAACAGATCATCCGCAAACAC and GTT GGGGCTCCTCAGGTTC) and p21 (GCAGACCAGCATG ACAGATTTC and CGGATTAGGGCTTCCTCTTG). The protocol was $95^{\circ} \mathrm{C}$ for $30 \mathrm{sec}, 40$ cycles of $95^{\circ} \mathrm{C}$ for $5 \mathrm{sec}$ and $60^{\circ} \mathrm{C}$ for $30 \mathrm{sec}$, and then a dissociation stage. The results were analyzed via the $\Delta \Delta \mathrm{Ct}$ method using GAPDH as the housekeeping gene.

Cell survival assay. Cells were treated with cisplatin (SigmaAldrich; 0-32 nmol/l) for $24 \mathrm{~h}$. In other experiments, the cells were irradiated with doses between 0 and 18 Gy under aerobic conditions, at room temperature, using a ${ }^{137} \mathrm{Cs}$ unit at a dose rate of $2.5 \mathrm{~Gy} / \mathrm{min}$.

Statistical analysis. All of the statistical analyses were performed using GraphPad Prism 5.0 software (GraphPad Software, La Jolla, CA, USA). All data are expressed as mean \pm standard deviation (SD). Student's t-test was used for comparisons between two groups and one-way or two-way analysis of variance (ANOVA) test was used to analyze statistical differences between groups under different conditions. $\mathrm{P}<0.05$ was considered to indicate a statistically significant difference. 
A
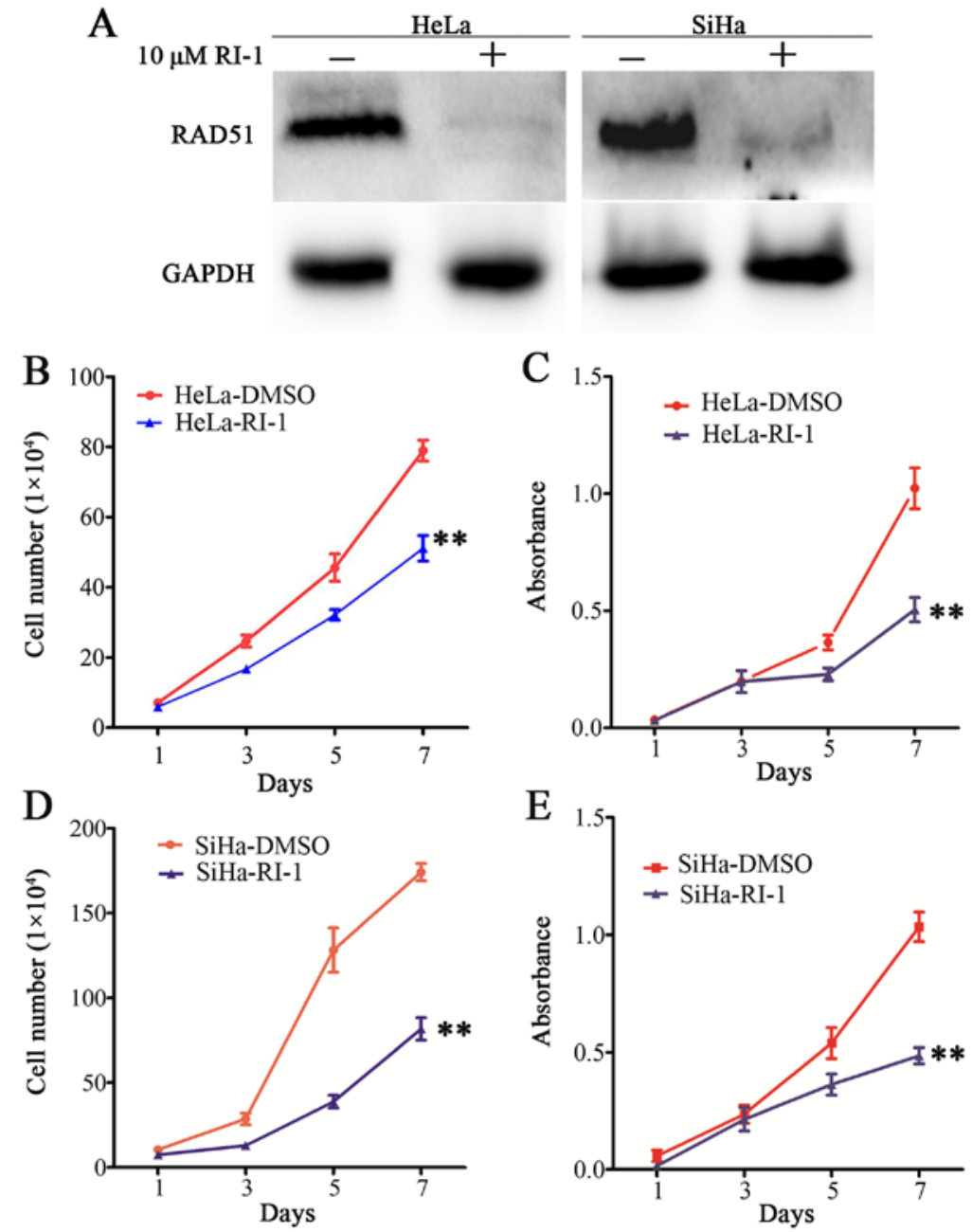

Figure 2. The RAD51 inhibitor RI-1 suppresses the proliferation of cervical cancer cells in vitro. (A) HeLa and SiHa cells were treated with either DMSO or $10 \mu \mathrm{M}$ RAD51 inhibitor RI-1 for $24 \mathrm{~h}$, and the RAD51 protein was measured by western blotting. Cell growth and MTT assays were performed using RI-1- or DMSO-treated HeLa cells (B and C) and RI-1- or DMSO-treated SiHa cells (D and E). The values are presented from independent experiments performed in triplicates as the mean $\pm \mathrm{SD}$. ${ }^{*} \mathrm{P}<0.05,{ }^{* *} \mathrm{P}<0.01$.

\section{Results}

RAD51 expression in normal cervix and squamous cervical carcinoma. To explore the RAD51 expression levels and its association with normal or neoplastic cervical tissues, we first conducted the immunohistochemistry assay using paraffinembedded normal cervix and squamous cervical cancer tissues. RAD51 staining was observed in the nuclei of positive cells in different cervical tissues (Fig. 1A). The number of specimens with positive RAD51 staining gradually increased from $13.95 \%$ (6/43) in the normal cervical tissues to $73.44 \%$ $(47 / 64)$ in the cervical cancer tissues (Table I and Fig. 1B, $\mathrm{P}<0.01$ ). IHC score results revealed that the immunoreactivity score (IRS) of RAD51 staining was 1.6 for the normal cervical tissues and 6.8 for the cervical cancer tissues $(\mathrm{P}<0.01$, Fig. 1C).

Next, using western blot assay, we found that RAD51 showed different expression levels in CaSki, C33-A, HeLa and SiHa cells (Fig. 1D). In particular, higher levels of the RAD51 protein appeared in the HeLa and Siha cells.

The RAD51 inhibitor RI-1 suppresses the proliferation of cervical cancer cells in vitro. Having shown that RAD51 expression was increased in cervical cancer progression, the role of RAD51 in cervical cancer was functionally evaluated. We conducted experiments using the RAD51 inhibitor RI-1. We found that RAD51 was almost eliminated using $10 \mu \mathrm{M}$ RI-1 in HeLa and SiHa cells that expressed higher levels of RAD51 protein by western blotting (Fig. 2A). Next, we tested whether the inhibition of RAD51 activity would suppress cervical cancer cell proliferation. Cell growth curve and MTT assays showed that compared to controls, RI-1 significantly reduced cell growth and viability in HeLa (Fig. $2 \mathrm{~B}$ and C, $\mathrm{P}<0.01$ ) and $\mathrm{SiHa}($ Fig. $2 \mathrm{D}$ and $\mathrm{E}, \mathrm{P}<0.01$ ). These results demonstrated that RI-1 suppressed the proliferation of cervical cancer cells in vitro.

The RAD51 inhibitor RI-1 suppresses the growth of cervical cancer xenografts in vivo. To investigate whether RI-1 has similar inhibitory effect on tumorigenicity in vivo, female athymic nude mice (6 mice per group) were injected subcutaneously with $\mathrm{HeLa}$ and $\mathrm{SiHa}$ cells. When tumor formation reached $120 \mathrm{~mm}^{3}$, the volume of tumors was measured every three days, and we intervened with an intraperitoneal injection of RI-1 (5 mg/kg in $200 \mu \mathrm{l}$ PBS) or normal saline. The net 

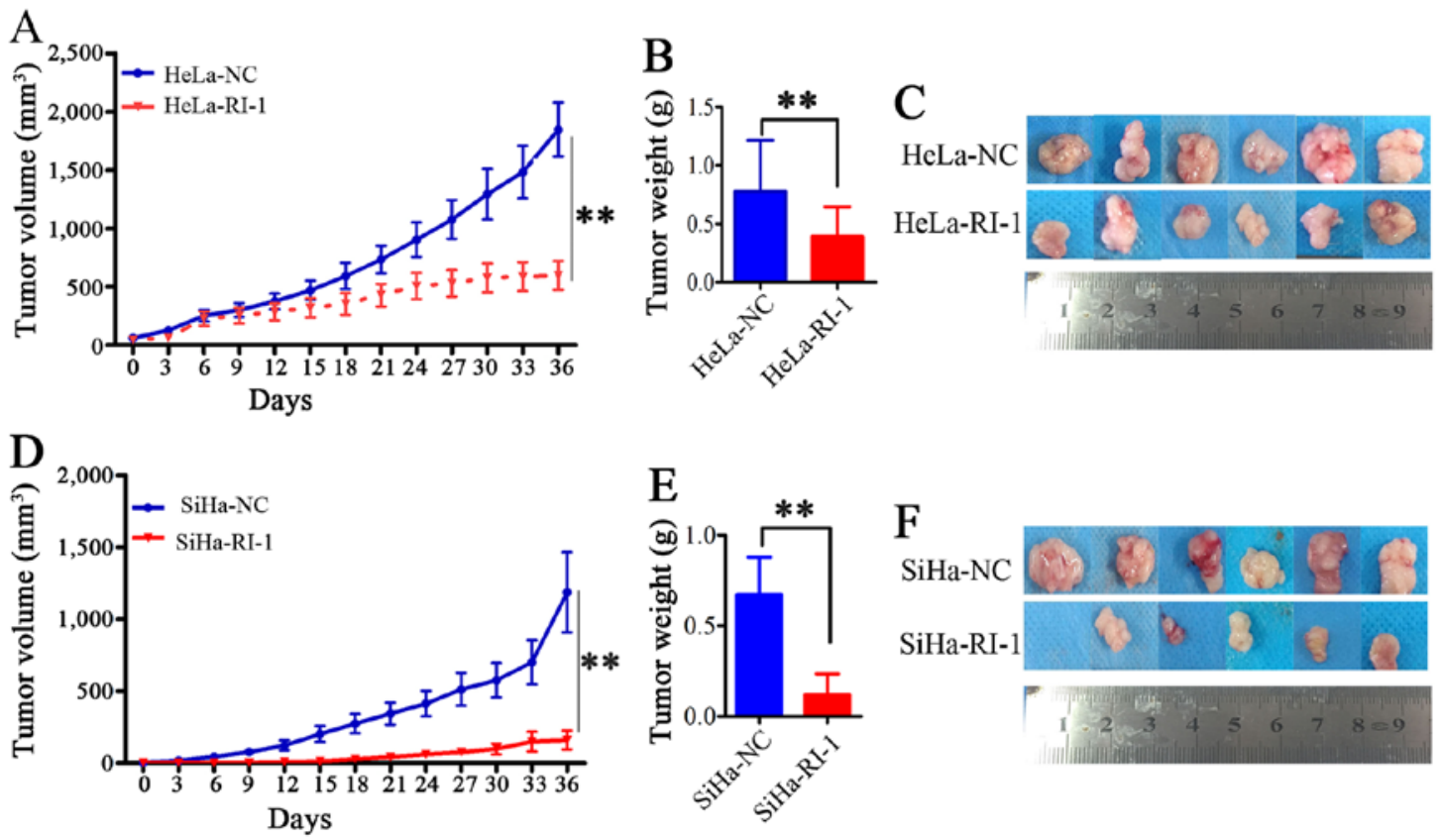

Figure 3. The RAD51 inhibitor RI-1 suppresses the growth of cervical cancer xenografts in vivo. (A-C) Tumor growth curves and tumor weights are shown for RI-1-treated HeLa and control cells. The NC group was treated with saline (n=6) and the RI-1 (n=6) group was treated with RI-1 for 36 days (intraperitoneal injection every other day). (D-F) Tumor growth curves and tumor weights are shown for RI-1-treated SiHa and control cells. The NC group was treated with saline $(n=6)$ and the RI-1 $(n=6)$ group with RI-1 for 36 days (intraperitoneal injection every other day). The values presented are of independent experiments performed in triplicates as the mean $\pm \mathrm{SD} .{ }^{*} \mathrm{P}<0.05,{ }^{* *} \mathrm{P}<0.01$.

weights of the sacrificed mice were recorded upon termination of the experiment. As shown in Fig. 3A, compared to the negative controls, the RI-1-treated mice exhibited significantly inhibited tumor growth in HeLa cells $(\mathrm{P}<0.01)$. The average volume and weight of xenografts of the RI-1-treated groups were markedly reduced (Fig. 3A-C, $\mathrm{P}<0.01$ ). The results were similar in SiHa cells (Fig. 3D-F, $\mathrm{P}<0.01$ ). These results indicated that RI-1 suppressed tumor formation of cervical cancer cells in vivo.

RI-1 suppresses the proliferation of cervical cancer cells by attenuating the cell cycle transition from G0/G1 to $S$ phase through acting on the expression of cell cycle associated protein cyclin D1 and p21. To investigate the mechanism of RI-1-mediated suppression of cervical cancer cell proliferation, cell cycle analyses of RI-1-HeLa and SiHa and control cells was performed by fluorescence-activated cell sorting (FACS). The percentage of cells in S phase significantly decreased from $47.25 \%$ for HeLa-DMSO cells to $31.56 \%$ for HeLa-RI-1 cells (Fig. 4A and B, P<0.05). A similar effect was observed in SiHa-RI-1 cells, which had $20.34 \%$ in the S phase compared to $33.12 \%$ of control cells (Fig. 4C and D, P<0.05). This result showed that the RAD51 inhibitor RI-1 may arrest the cell cycle transition at the G1/S phase and further suppress the proliferation of cervical cancer cells.

Further experiments showed that the mRNA level of the cell cycle associated protein cyclin D1 was reduced while that of p21 increased after RI-1 treatment in HeLa and SiHa cells (Fig. 4E, $\mathrm{P}<0.05$ ). The administration of RI-1 for $48 \mathrm{~h}$ resulted in a downregulation of cyclin D1 and upregulation of $\mathrm{p} 21$ by western blotting (Fig. 4F, $\mathrm{P}<0.01$ ). Collectively, these results suggested that inhibition of RAD51 blocked the cell cycle transition possibly through interaction with cyclin D1 and p21.
Inhibition of RAD51 enhances the sensitivity of cervical cancer cells to chemotherapeutic drugs and irradiation. Given the decreased expression and function of RAD51 in cervical cancer cell lines after using RI-1, we investigated whether RI-1 could lead to an increase in tumor cell sensitivity to cisplatin and radiation. We observed that cervical cancer cells, HeLa and SiHa, when treated with RI-1 had significantly reduced survival compared to cells treated with DMSO in response to cisplatin (Fig. 5A and B). In response to radiotherapy, HeLa and $\mathrm{SiHa}$ cells treated with RI-1 had also significantly reduced survival compared to cells treated with DMSO (Fig. 5C and D). Thus, we concluded that RI-1 can sensitize cervical cancer cells to chemotherapeutic drugs and radiation.

\section{Discussion}

Elevated expression of RAD51 is associated with tumor aggressiveness and is known to confer treatment resistance in a variety of tumors, including those in ovarian cancer (19), breast cancer (16), lung tumors (20), pancreatic adenocarcinoma (13) and malignant gliomas (21). Furthermore, downregulation of RAD51 protein by RAD51 antisense oligonucleotides, RNA interference (22), aptamers (23) or small molecule inhibitors against RAD51 could be used to sensitize tumors to chemotherapy or radiation. Recent studies have identified that impaired replication and intra-S mediated CHK1 signaling by RAD51 led to higher genomic instability and thus drove tumorigenesis (24). The 3'-untranslated region of RAD51 was directly bound by tumor-suppressing miR-34a and thus regulation of homologous recombination and double-strand break repair was inhibited in NSCLC cells (20).

In our study, RAD51 expression was found to be significantly elevated in cervical cancer tissues compared to normal 
A

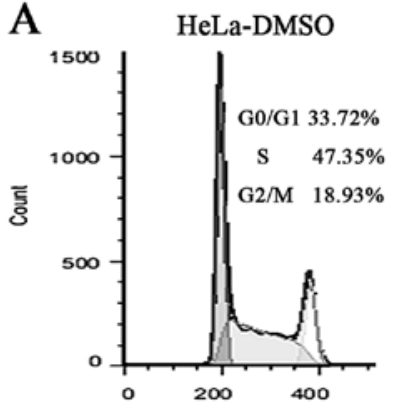

C

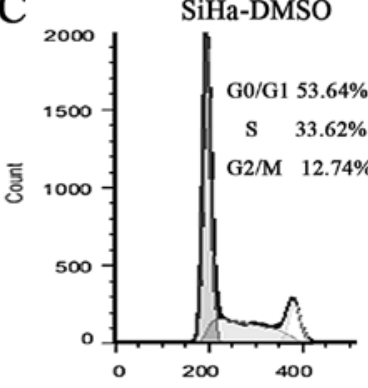

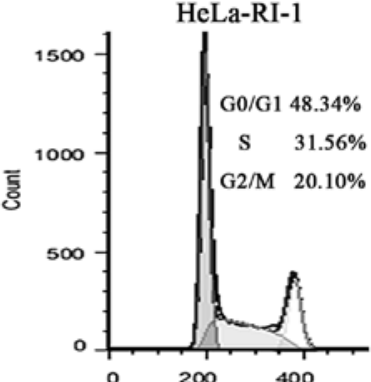
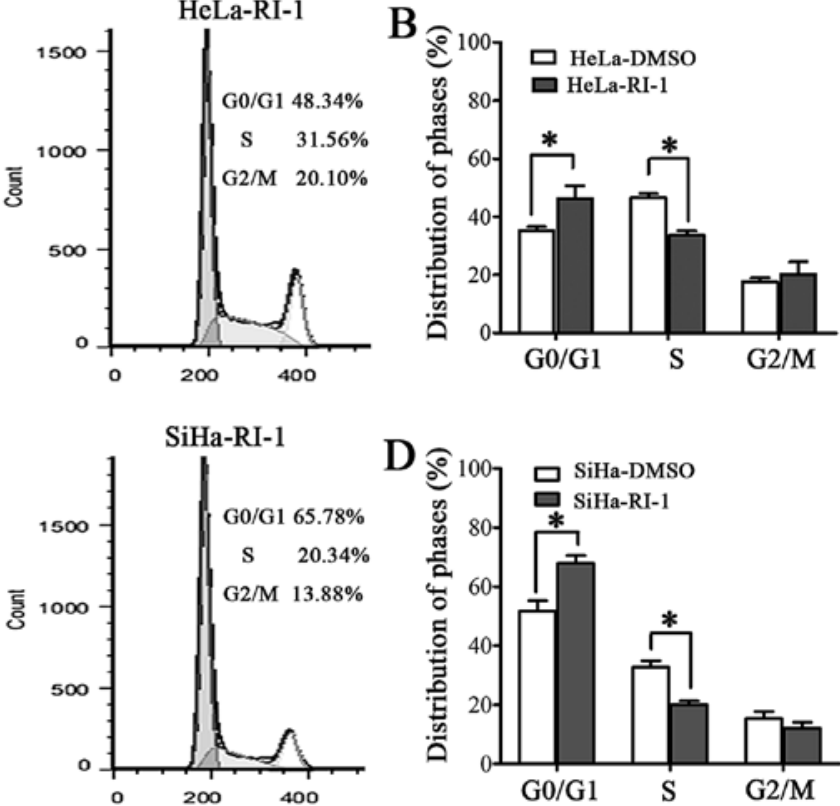

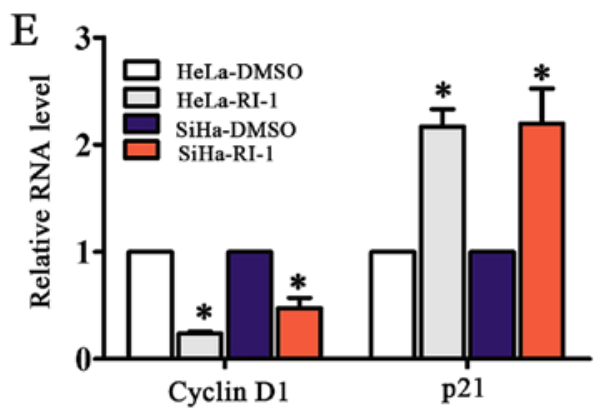

F
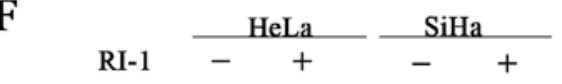

Cyclin D1

p21

GAPDH

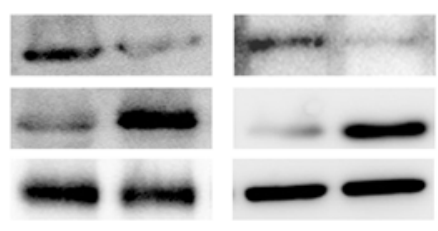

Figure 4. The RAD51 inhibitor RI-1 suppresses the proliferation of cervical cancer cells by attenuating the cell cycle transition from G0/G1 to S phase through its effect on the expression of cell cycle associated protein cyclin D1 and p21. Cell cycle progression was analyzed by FACS, and the results of quantitative analysis of the cell cycle distribution are shown for RI-1-treated HeLa cells and control cells (A and B) and RI-1-treated SiHa cells and control cells (C and D). (E) The relative mRNA levels of cyclin D1 and p21 in RI-1-treated HeLa and SiHa cells as determined by quantitative real-time PCR. (F) The expression of cyclin D1 and p21 in RI-1-treated HeLa and SiHa cells was determined by western blotting. The values presented are from independent experiments performed in triplicates as the mean $\pm \mathrm{SD} .{ }^{*} \mathrm{P}<0.05,{ }^{* *} \mathrm{P}<0.01$.
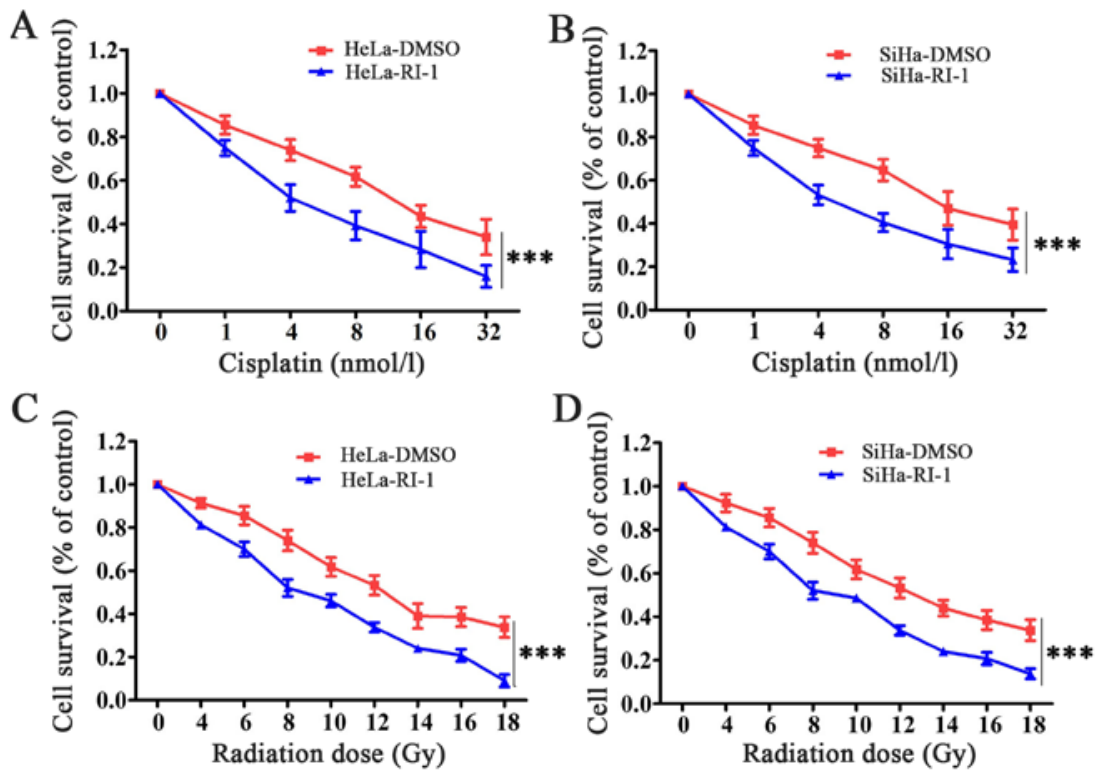

Figure 5. The RAD51 inhibitor RI-1 enhances the sensitivity of cervical cancer cells to chemotherapeutic drugs and irradiation. (A) RI-1-treated HeLa and (B) SiHa cells were treated with different concentrations of cisplatin for $24 \mathrm{~h}$ and subjected to MTT assay. (C) RI-1-treated HeLa and (D) SiHa cells were irradiated with the indicated doses for $24 \mathrm{~h}$ and evaluated with MTT assay. The mean survival for three independent experiments are shown. The values presented are from independent experiments performed in triplicates as the mean $\pm \mathrm{SD}$. ${ }^{*} \mathrm{P}<0.05,{ }^{* * *} \mathrm{P}<0.01 .{ }^{* * *} \mathrm{P}<0.001$. 
cervical tissues (Fig. 1), consistent with results from previous studies of non-small cell lung cancer (7), breast carcinoma (8), prostate cancer (25), chronic myeloid leukaemia (CML), melanoma and glioblastoma (11). Subsequently, RI-1 was applied to further explore the function of RAD51 in cervical carcinogenesis. RI-1 could directly and specifically disrupt HsRAD51, and inhibit sub-nuclear accumulation of HsRAD51 protein at sites of DNA damage, thereby, this inhibitory activity sensitizes tumor cells to cross-linking chemotherapy (15). We further performed western blotting to evaluate the inhibitory effect of RI-1 on RAD51. A previous study indicated that the combination of minocycline and MMC in NSCLC can synergistically inhibit cell proliferation and reduce cell viability in vitro, while overexpression of RAD51 expression can restore cell viability upon minocycline and MMC cotreatment (26). In our study, the results showed that inhibition of RAD51 significantly suppressed the proliferation of cervical cancer cells in vitro and tumor growth in vivo (Figs. 2 and 3). In a previous study, p21 was investigated because it was well-known as a major mediator of G1 arrest and an inhibitor of G1 Cdks after DNA damage (27). Thus, the RAD51 foci-positive cells could also be arrested during the G1 phase. This would be consistent with the observation that G0/G1 arrest upon serum starvation induced by overexpressed of RAD51 protein. However, endogenous Rad51 foci were not observed in G1 phase cells. Overexpression of RAD51 could increase its function, which improved DNA recombination in S/G2 phase (28). Our cell cycle analyses suggested that RI-1 prevented the transition from $\mathrm{G} 0 / \mathrm{G} 1$ to $\mathrm{S}$ phase in cervical cancer cells. In addition, RI-1 treatment decreased cyclin D1 and increased p21 mRNA and protein levels (Fig. 4). Nevertheless, the particular mechanism between RAD51 and cyclin D1 and p21 should be further investigated. Recent studies have revealed that the enhanced RAD51 protein level in tumor cells is associated with high DNA repair capacity, elevated recombination rates and increased resistance against radiotherapy and chemotherapy (10). Our study showed that downregulation of RAD51 protein could indeed sensitize cervical cancer cells to chemotherapy and radiation (Fig. 5).

In summary, our findings demonstrate that inhibition of RAD51 attenuates cell proliferation and tumor formation in cervical cancer cells by arresting the cell cycle via cyclin D1 and p21. Therefore, RAD51 might be a promising therapeutic strategy for the treatment of cervical cancer.

\section{Acknowledgements}

This work was supported by a grant from the Science and Technology Project of Shaanxi Province (2014K11-01-01-18).

\section{References}

1. Torre LA, Bray F, Siegel RL, Ferlay J, Lortet-Tieulent J and Jemal A: Global cancer statistics, 2012. CA Cancer J Clin 65: $87-108,2015$

2. Walboomers JM, Jacobs MV, Manos MM, Bosch FX, Kummer JA, Shah KV, Snijders PJ, Peto J, Meijer CJ and Muñoz N: Human papillomavirus is a necessary cause of invasive cervical cancer worldwide. J Pathol 189: 12-19, 1999.

3. Zhang L, Wu J, Ling MT, Zhao L and Zhao KN: The role of the $\mathrm{PI} 3 \mathrm{~K} / \mathrm{Akt} / \mathrm{mTOR}$ signalling pathway in human cancers induced by infection with human papillomaviruses. Mol Cancer 14: 87, 2015 .
4. Qureshi R, Arora H and Rizvi MA: EMT in cervical cancer: Its role in tumour progression and response to therapy. Cancer Lett 356: 321-331, 2015.

5. Kowalczykowski SC: Biochemistry of genetic recombination Energetics and mechanism of DNA strand exchange. Annu Rev Biophys Biophys Chem 20: 539-575, 1991.

6. Paulíková S, Chmelařová M, Petera J, Palička V and Paulík A Hypermethylation of RAD51L3 and XRCC2 genes to predict late toxicity in chemoradiotherapy-treated cervical cancer patients. Folia Biol (Praha) 59: 240-245, 2013.

7. Takenaka T, Yoshino I, Kouso H, Ohba T, Yohena T, Osoegawa A, Shoji F and Maehara Y: Combined evaluation of Rad51 and ERCC1 expressions for sensitivity to platinum agents in non-small cell lung cancer. Int J Cancer 121: 895-900, 2007.

8. Maacke H, Opitz S, Jost K, Hamdorf W, Henning W, Krüger S, Feller AC, Lopens A, Diedrich K, Schwinger E, et al: Overexpression of wild-type Rad51 correlates with histological grading of invasive ductal breast cancer. Int J Cancer 88: 907-913, 2000.

9. Liu G, Yang D, Rupaimoole R, Pecot CV, Sun Y, Mangala LS, Li X, Ji P, Cogdell D, Hu L, et al: Augmentation of response to chemotherapy by microRNA-506 through regulation of RAD51 in serous ovarian cancers. J Natl Cancer Inst 107: 107, 2015.

10. Maacke H, Jost K, Opitz S, Miska S, Yuan Y, Hasselbach L, Lüttges J, Kalthoff $\mathrm{H}$ and Stürzbecher HW: DNA repair and recombination factor Rad51 is over-expressed in human pancreatic adenocarcinoma. Oncogene 19: 2791-2795, 2000.

11. Raderschall E, Stout K, Freier S, Suckow V, Schweiger S and Haaf T: Elevated levels of Rad51 recombination protein in tumor cells. Cancer Res 62: 219-225, 2002.

12. Hine CM, Seluanov A and Gorbunova V: Use of the Rad51 promoter for targeted anti-cancer therapy. Proc Natl Acad Sci USA 105: 20810-20815, 2008.

13. Nagathihalli NS and Nagaraju G: RAD51 as a potential biomarker and therapeutic target for pancreatic cancer. Biochim Biophys Acta 1816: 209-218, 2011.

14. Wang AT, Kim T, Wagner JE, Conti BA, Lach FP, Huang AL, Molina $\mathrm{H}$, Sanborn EM, Zierhut $\mathrm{H}$, Cornes BK, et al: A dominant mutation in human RAD51 reveals its function in DNA interstrand crosslink repair independent of homologous recombination. Mol Cell 59: 478-490, 2015.

15. Budke B, Logan HL, Kalin JH, Zelivianskaia AS, Cameron McGuire W, Miller LL, Stark JM, Kozikowski AP, Bishop DK and Connell PP: RI-1: A chemical inhibitor of RAD51 that disrupts homologous recombination in human cells. Nucleic Acids Res 40: 7347-7357, 2012.

16. Hong KJ, Hsu MC and Hung WC: RECK impedes DNA repair by inhibiting the erbB/JAB1/Rad51 signaling axis and enhances chemosensitivity of breast cancer cells. Am J Cancer Res 5: 2422-2430, 2015

17. Choudhury A, Zhao H, Jalali F, Al Rashid S, Ran J, Supiot S, Kiltie AE and Bristow RG: Targeting homologous recombination using imatinib results in enhanced tumor cell chemosensitivity and radiosensitivity. Mol Cancer Ther 8: 203-213, 2009.

18. Kübler HR, van Randenborgh H, Treiber U, Wutzler S, Battistel C, Lehmer A, Wagenpfeil S, Hartung R and Paul R: In vitro cytotoxic effects of imatinib in combination with anticancer drugs in human prostate cancer cell lines. Prostate 63: 385-394, 2005

19. Wang B, Hou D, Liu Q, Wu T, Guo H, Zhang X, Zou Y, Liu Z, Liu J, Wei J, et al: Artesunate sensitizes ovarian cancer cells to cisplatin by downregulating RAD51. Cancer Biol Ther 16: 1548-1556, 2015.

20. Cortez MA, Valdecanas D, Niknam S, Peltier HJ, Diao L, Giri U, Komaki R, Calin GA, Gomez DR, Chang JY, et al: In vivo delivery of miR-34a sensitizes lung tumors to radiation through RAD51 regulation. Mol Ther Nucleic Acids 4: e270, 2015.

21. Ohnishi T, Taki T, Hiraga S, Arita N and Morita T: In vitro and in vivo potentiation of radiosensitivity of malignant gliomas by antisense inhibition of the RAD51 gene. Biochem Biophys Res Commun 245: 319-324, 1998

22. Mueller AC, Sun D and Dutta A: The miR-99 family regulates the DNA damage response through its target SNF2H. Oncogene 32: 1164-1172, 2013.

23. Martinez SF, Renodon-Cornière A, Nomme J, Eveillard D, Fleury F, Takahashi M and Weigel P: Targeting human Rad51 by specific DNA aptamers induces inhibition of homologous recombination. Biochimie 92: 1832-1838, 2010. 
24. Parplys AC, Seelbach JI, Becker S, Behr M, Wrona A, Jend C, Mansour WY, Joosse SA, Stuerzbecher HW, Pospiech H, et al High levels of RAD51 perturb DNA replication elongation and cause unscheduled origin firing due to impaired CHK1 activation. Cell Cycle 14: 3190-3202, 2015.

25. Mitra A, Jameson C, Barbachano Y, Sanchez L, Kote-Jarai Z Peock S, Sodha N, Bancroft E, Fletcher A, Cooper C, et al; IMPACT Steering Committee and IMPACT and EMBR ACE Collaborators: Overexpression of RAD51 occurs in aggressive prostatic cancer. Histopathology 55: 696-704, 2009.

26. Ko JC, Wang TJ, Chang PY, Syu JJ, Chen JC, Chen CY, Jian YT, Jian YJ, Zheng HY, Chen WC, et al: Minocycline enhances mitomycin $\mathrm{C}$-induced cytotoxicity through down-regulating ERK1/2-mediated Rad51 expression in human non-small cell lung cancer cells. Biochem Pharmacol 97: 331-340, 2015.
27. Deng C, Zhang P, Harper JW, Elledge SJ and Leder P: Mice lacking p21CIP1/WAF1 undergo normal development, but are defective in G1 checkpoint control. Cell 82: 675-684, 1995.

28. Tashiro S, Walter J, Shinohara A, Kamada N and Cremer T: Rad51 accumulation at sites of DNA damage and in postreplicative chromatin. J Cell Biol 150: 283-291, 2000. 